\title{
RECONSTRUCCIÓN MAMARIA: ESTADO ACTUAL DEL TEMA*
}

\author{
Drs. Diego Marré ${ }^{1}$ José Tomás Gantz ${ }^{2}$ Javier Villalón ${ }^{2,3}$, Héctor Roco ${ }^{3,4}$ \\ 1 Departamento de Cirugía Plástica, Clínica Universidad de Navarra, Navarra, España. \\ 2 Unidad de Patología Mamaria, Cirugía Plástica, Hospital Padre Hurtado, Santiago, Chile. \\ 3 Servicio de Cirugía Plástica, Clínica Alemana de Santiago, Santiago, Chile. \\ ${ }^{4}$ Servicio de Cirugía Plástica, Hospital de la Fuerza Aérea de Chile, Santiago, Chile.
}

\begin{abstract}
\section{Breast reconstruction: state of the art}

Nowadays most plastic surgery units worldwide perform breast reconstruction and hence basic updated knowledge in the topic is mandatory for every plastic surgeon and trainee. Breast reconstruction may be performed either immediately after mastectomy or in a delayed fashion. Each of these timings has its advantages and drawbacks and albeit there are a number of factors influencing the decision to perform one or another, probably the most important one has to do with the delivery of radiotherapy. In this sense, while in patients who are candidates for post mastectomy radiation, a delayed reconstruction is strongly recommended, in those without adjuvancy, immediate reconstruction is a very good alternative. In general terms, breast reconstruction techniques fall into one of three groups: those using alloplastic materials only; those performed with autologous tissue only; and those using a combination of both alloplastics and autologous tissue. Again the administration of radiotherapy, as well as patient and surgeon-related factors, play a fundamental role when choosing anyone technique. Finally, during the last years there has been growing interest and evidence on the use of acellular dermal matrices for breast reconstruction, which have expanded the armamentarium of techniques available for these patients. The following paper aims to provide an updated review on breast reconstruction regarding timing of reconstruction, techniques available, the influence of radiotherapy and the use of acellular dermal matrices.
\end{abstract}

Key words: Breast cancer, mastectomy, breast reconstruction, acellular dermal matrices.

\section{Resumen}

La reconstrucción mamaria constituye una parte importante de la práctica de muchos servicios y unidades de cirugía plástica y por tanto resulta muy necesario que tanto cirujanos plásticos con experiencia como aquellos en formación tengan conocimientos básicos actualizados sobre el tema. La reconstrucción mamaria puede llevarse a cabo de forma inmediata después de la mastectomía o de forma diferida. Cada una de estas modalidades tiene ventajas y desventajas. Si bien existen distintos factores para elegir uno u otro timing, la

*Recibido el 1 de julio de 2015 y aceptado para publicación el 19 de julio de 2015.

Conflictos de interés: ninguno

Correspondencia: Dr. Diego Marré

diegomarre@gmail.com 
necesidad de radioterapia postmastectomía es probablemente el factor más importante a considerar. De esta manera, en términos generales, se recomienda realizar una reconstrucción diferida en pacientes subsidiarias de irradiación, mientras que en pacientes sin indicación de dicha adyuvancia, la reconstrucción inmediata es una muy buena alternativa. Dentro de las técnicas de reconstrucción, existen tres grandes grupos: las que utilizan materiales aloplásticos únicamente; las que utilizan tejidos autólogos; y las que usan ambos elementos. La decisión de cuál técnica utilizar estará influenciada por factores tanto de la paciente como del cirujano y el centro hospitalario. Durante los últimos años ha habido un interés creciente en el uso de matrices dérmicas acelulares en reconstrucción mamaria que han permitido ampliar el arsenal de alternativas terapéuticas que ofrecer a las pacientes mastectomizadas. El siguiente trabajo tiene por objetivo presentar una revisión actualizada de la literatura sobre reconstrucción mamaria en cuanto a tiempos de reconstrucción, técnicas más frecuentes, influencia de la radioterapia y el uso de matrices dérmicas acelulares.

Palabras clave: Cáncer de mama, mastectomía, reconstrucción, matrices dérmicas acelulares.

\section{Introducción}

La reconstrucción mamaria constituye una parte importante de la práctica de muchos servicios y unidades de cirugía plástica. Los avances tecnológicos y el refinamiento de algunas técnicas de reconstrucción, así como la descripción de nuevos procedimientos, permiten actualmente contar con múltiples alternativas terapéuticas que ofrecer a las pacientes mastectomizadas. El uso de implantes y colgajos son técnicas que solas o combinadas han demostrado tener un resultado predecible y estéticamente muy satisfactorio. Sumado a esto, durante los últimos años ha habido un creciente interés y desarrollo en el uso de matrices dérmicas con las cuales se han abierto nuevas opciones de reconstrucción.

En el siguiente trabajo presentamos una revisión general actualizada sobre reconstrucción mamaria en cuanto al timing, las técnicas más frecuentes, la influencia de la radioterapia y el uso de matrices dérmicas acelulares.

\section{Epidemiología del cáncer de mama}

La Organización Mundial de la Salud, a través de su Agencia Internacional en el Estudio del Cáncer (IARC), estimó una incidencia mundial de 1.676.632 casos de cáncer de mama en 2012, con una mortalidad de 12,9 por 100.000 mujeres. En el análisis por región, se observaron 499.555 nuevos casos en Europa y 408.266 en América y el Caribe. Según el mismo estudio, Chile presentó un total de 4.081 nuevos $\operatorname{casos}^{1}$. Por otra parte, registros nacionales del MINSAL al año 2011 muestran una tasa de mortalidad de 15,46 por 100.000 mujeres. Respecto al estadio, sólo el $10 \%$ de los casos se diagnosticó en estadio 0 y el $16,7 \%$ en estadío I; la mayoría (42\%) de los casos fueron diagnosticados en estadio $\mathrm{II}^{2}$.

Si bien el tratamiento quirúrgico del cáncer de mama ha migrado en las últimas décadas hacia téc- nicas que conservan el tejido mamario, la mastectomía continúa siendo un procedimiento frecuente. Sin embargo, la reconstrucción mamaria aún no constituye un tratamiento al alcance de la gran mayoría de las pacientes mastectomizadas. Estudios realizados en los centros designados por el Instituto Nacional del Cáncer de EE. UU muestran que menos del $50 \%$ de las pacientes mastectomizadas son reconstruidas ${ }^{3}$.

Respecto del tiempo de reconstrucción, un estudio basado en el programa de registros del Instituto Nacional del Cáncer Americano (SEER: Surveillance, Epidemiology, and End Results) describe que del total de pacientes que completaron el seguimiento del estudio, el 24,8\% fueron reconstruidas de forma inmediata y el $16,8 \%$ de forma diferida ${ }^{4}$.

En relación a la técnica de reconstrucción, de un total de 91.655 reconstrucciones reportadas en 2012 por la Sociedad Americana de Cirujanos Plásticos, un $8,1 \%$ fueron realizados utilizando sólo implantes; un $70,5 \%$ con la técnica de expansor prótesis; un $6,6 \%$ con colgajo TRAM; y un $7,1 \%$ con colgajo DIEP $^{5}$

A nivel nacional, actualmente no se cuenta con un registro confiable de reconstrucción mamaria en cualquiera de sus formas y tiempos, tanto en el sector público como privado.

\section{Reconstrucción mamaria: inmediata vs diferida}

La primera pregunta a contestar frente a una paciente afectada de cáncer de mama que será sometida a mastectomía es si realizar la reconstrucción de forma inmediata tras la resección oncológica o de forma diferida. Dado que no existen estudios concluyentes sobre la superioridad de uno u otro timing, la recomendación general es evaluar cada caso de forma individual ${ }^{6,7}$, ya que cada uno tiene ventajas concretas las cuales se resumen en la Tabla $1^{8,9}$. 
Tabla 1. Ventajas de reconstrucción mamaria inmediata y diferida

\begin{tabular}{|c|c|}
\hline Inmediata & Diferida \\
\hline - Ausencia de cicatriz, fibrosis y/o retracción de los tejidos & $\begin{array}{l}\text { Evita los problemas asociados a la radioterapia, tanto } \\
\text { en una reconstrucción con implantes como con tejidos } \\
\text { propios }\end{array}$ \\
\hline - Preservación del surco submamario & $\begin{array}{l}\text { Es posible completar el estudio histopatológico, y por } \\
\text { tanto definir la necesidad de tratamiento adyuvante, antes } \\
\text { de la reconstrucción }\end{array}$ \\
\hline $\begin{array}{l}\text { - El bolsillo cutáneo una vez rellenado vuelve a su forma } \\
\text { y proyección natural, lo cual se traduce en una mejor } \\
\text { simetría con el lado sano }\end{array}$ & $\begin{array}{l}\text { - Se evitan complicaciones relacionadas con el sufrimiento } \\
\text { vascular de los colgajos de mastectomía }\end{array}$ \\
\hline $\begin{array}{l}\text { - Al conseguir una mayor simetría se reduce la necesidad } \\
\text { de cirugía en la mama sana, con lo cual la reconstrucción } \\
\text { puede hacerse en muchos casos en un solo tiempo y por } \\
\text { tanto con un menor costo económico }\end{array}$ & \\
\hline $\begin{array}{l}\text { - Beneficio psicológico para la paciente que en ningún } \\
\text { momento del proceso se ve a si misma mastectomizada }\end{array}$ & \\
\hline
\end{tabular}

La principal desventaja de la reconstrucción inmediata es el daño a los tejidos que puede producir una radioterapia inesperada, mientras que las de la reconstrucción diferida incluyen la necesidad de contar con una isla cutánea para la reconstrucción (en caso que haya habido daño por radioterapia) cuyo resultado estético es inferior al de la piel original de la mama; la necesidad de una segunda cirugía aparte de la mastectomía; y el estrés psicológico de la paciente que se ve mastectomizada ${ }^{8}$.

Si bien la influencia de la radioterapia en la elección de la técnica de reconstrucción será abordado con mayor detalle más adelante, a estas alturas es necesario comentar que en general se recomienda realizar una reconstrucción diferida en pacientes subsidiarias de radioterapia postmastectomía debido a los efectos deletéreos que este tratamiento tiene sobre los tejidos y en el resultado global de la reconstrucción ${ }^{10}$. Por el contrario, cuando se tiene certeza de que no habrá radiación, es recomendable proceder con la reconstrucción inmediata debido a las ventajas antes citadas. Sin embargo, existe un grupo de pacientes en las que la indicación de radioterapia sólo se sabe una vez finalizado el estudio histopatológico de la pieza de mastectomía y/o el(los) ganglio(s) centinela resecado(s). El dilema en estos casos es que si no se realiza una reconstrucción inmediata y finalmente no hay necesidad de radioterapia, se priva a la paciente de los beneficios de dicho timing. Por el contrario, si se procede con la reconstrucción y la paciente es irradiada, la probabilidad de complicaciones y/o de obtener un resultado subóptimo se incrementan. Como solución a esto se describió la reconstrucción diferida-inmediata

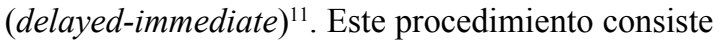
en colocar un expansor subpectoral al momento de la mastectomía y realizar una expansión intraoperatoria para conservar la forma y dimensiones del bolsillo cutáneo. Con los resultados definitivos de la histopatología, aquellas pacientes que no van a recibir radioterapia prosiguen con la reconstrucción (con implantes o autóloga dependiendo del caso) mientras que aquellas subsidiarias de ser irradiadas, permanecen con el expansor el cual es vaciado totalmente previo al tratamiento. Una vez finalizada la radiación, el expansor es rellenado nuevamente para luego proceder con la reconstrucción autóloga cuatro a seis meses después.

\section{Técnicas de reconstrucción: implantes y tejidos autólogos}

Las técnicas de reconstrucción mamaria pueden agruparse de forma genérica según si se basan en el uso de materiales aloplásticos (expansores e implantes mamarios), si utilizan únicamente tejidos autólogos, o una combinación de ambas. La decisión de realizar una u otra dependerá de una serie de factores como la presencia de radioterapia, características físicas, preferencias y expectativas de la paciente, preferencia y experiencia del cirujano y disponibilidad de la infraestructura adecuada en el centro hospitalario.

\section{i) Reconstrucción con implantes: prótesis directa y expansor-prótesis}

La reconstrucción mamaria con implantes es la técnica más frecuentemente utilizada en Chile así 
como en el resto del mundo 5 . Si bien puede realizarse en prácticamente cualquier paciente, las mejores candidatas a este tipo de reconstrucción son aquellas con mamas de tamaño pequeño-mediano y con un bajo grado de ptosis ${ }^{12}$. En términos generales, la técnica de prótesis directa estaría indicada en pacientes con mamas pequeñas donde existe piel y músculo suficientes con los que cubrir la gran mayoría de la superficie protésica. Si esto no es posible, se debe recurrir a la expansión. Las grandes ventajas de estas técnicas son su relativa simplicidad, menor tiempo quirúrgico $\mathrm{y}$ ausencia de morbilidad en zona donante. Por el contrario, las desventajas incluyen el llevar un cuerpo extraño, las complicaciones derivadas del uso de implantes como son la contaminación, exposición, infección, contractura capsular, desplazamiento, ruptura, la probabilidad de necesitar un recambio de los implantes en algún momento de la evolución y, en caso de reconstrucción unilateral, el hecho que la mama reconstruida adquiere una posición y proyección relativamente fijas y progresivamente asimétrica en relación con la mama contralateral.

\section{ii) Reconstrucción con tejido autólogo}

Este grupo de técnicas se basa en la restauración del volumen y contorno mamario utilizando tejidos de la propia paciente, lo cual tiene como grandes ventajas la ausencia de cuerpos extraños y eliminación de las potenciales complicaciones asociadas al uso de implantes. Por otra parte, el tejido transferido tiene un comportamiento más acorde con la evolución y variaciones del peso y contextura física de la paciente y la consistencia de la mama reconstruida es más natural que aquella obtenida con implantes. Como desventajas, la reconstrucción con colgajos es técnicamente más difícil, requiere de mayor tiempo operatorio, existe morbilidad en la zona donante y generalmente requiere también de mayor tiempo de hospitalización y recuperación. Clásicamente, el colgajo musculocutáneo de recto abdominal (transverse rectus abdominis myocutaneous, TRAM) ha sido la técnica de elección para reconstrucción mamaria autóloga en muchos centros ${ }^{13,14}$. Tratándose de un músculo tipo III en la clasificación de Mathes y Nahai, el colgajo TRAM puede basarse tanto en los vasos epigástricos superiores (TRAM pediculado) como en los epigástricos inferiores profundos (TRAM libre microquirúrgico). La principal desventaja del colgajo TRAM es la debilidad que produce en la pared abdominal con el potencial riesgo de limitación funcional, bulging y desarrollo de hernias ${ }^{15}$. Como solución a esto, se han descrito refinamientos de la técnica destinados a reducir la morbilidad abdominal, como son el TRAM ahorrador de músculo I y II (muscle-sparing TRAM), el colgajo de perforantes de la arteria epigástrica inferior profunda (deep inferior epigastric artery perforator flap, DIEP) y el colgajo de arteria epigástrica inferior superficial (superficial inferior epigastric artery flap, SIEA). Mientras que con el muscle-sparing se sacrifica sólo una parte de músculo, con el DIEP se minimiza la morbilidad ya que las fibras musculares no son resecadas sino separadas preservando la inervación. Este último colgajo es actualmente la técnica de elección para reconstrucción autóloga en muchos centros. Estudios comparativos han demostrado una tendencia hacia una menor morbilidad abdominal con el DIEP en comparación con el TRAM y MSTRAM $^{16}$. Con el SIEA en cambio, dicha morbilidad se elimina ya que la disección del colgajo es completamente suprafascial. Sin embargo, hay que tener en cuenta que la arteria epigástrica superficial está ausente en un $35-40 \%$ de las pacientes y que cuando existe, no siempre es de un calibre adecuado (mayor a $1,5 \mathrm{~cm}$ ) con lo cual el uso de este colgajo es más limitado ${ }^{17}$. Las principales desventajas del DIEP en comparación con el TRAM son su mayor dificultad técnica, una curva de aprendizaje más pronunciada y la necesidad de contar con infraestructura microquirúrgica (a diferencia del TRAM pediculado), lo cual no es posible en todos los centros. Otros colgajos utilizados, todos ellos microquirúrgicos, son los basados en perforantes de las arterias glúteas superior (SGAP) e inferior (IGAP) y el colgajo musculocutáneo transverso de gracilis (TMG). Generalmente, estos colgajos constituyen la segunda opción en caso de no poder realizar un DIEP. El SGAP/IGAP tiene como desventajas una mayor dificultad técnica en la disección de la perforante, la necesidad de un cambio de posición dado que deben ser elevados en decúbito prono, el riesgo de alteración del contorno corporal en la zona donante y el hecho que el tejido glúteo es más fibroso y compacto que el abdominal con lo cual la mama reconstruida resulta menos natural $^{18}$. El TMG resulta una muy buena alternativa cuando no existe tejido suficiente a nivel abdominal o glúteo, aunque hay grupos para los cuales constituye la técnica de elección en algunos $\operatorname{casos}^{19}$. Su principal limitación tiene que ver con las posibles secuelas en la zona donante y su volumen limitado de piel y grasa $^{20}$. Un colgajo relativamente nuevo en el arsenal de reconstrucción mamaria es el basado en perforantes de la arteria femoral profunda llamado colgajo PAP (profunda artery perforator) el cual constituye una alternativa más al abdomen ${ }^{21}$.

\section{iii) Reconstrucción con tejido autólogo + implantes}

Este grupo de técnicas se basa en reconstruir el volumen y contorno mamario con ambos elementos. Generalmente, el tejido autólogo cumple una 
función de cobertura, protección y aportación de piel ausente, mientras que el implante otorga el volumen y la forma. Si bien es cierto que se puede asociar una prótesis a cualquier colgajo, el dorsal ancho (muscular o musculocutáneo) es sin duda el más empleado en esta modalidad. Este colgajo aporta una gran cantidad de músculo, una isla cutánea considerable y posee una vascularización segura y constante. La repercusión funcional es variable y generalmente más notoria en pacientes deportistas ${ }^{22}$. La complicación más frecuente de este colgajo es el seroma de la zona donante dada la amplia disección que se realiza.

\section{Influencia de la radioterapia en reconstruc- ción mamaria}

Las indicaciones clásicas de radioterapia en cáncer de mama incluyen tumores T3 o T4 $(\geq 5 \mathrm{~cm})$ y 4 o más ganglios afectados ${ }^{23}$. Sin embargo, en estudios recientes se ha observado un posible beneficio en estadios más tempranos con 1 a 3 ganglios afectados ${ }^{24}$. Desde el punto de vista reconstructivo, este hecho es de gran relevancia dado que, en general, cualquier tipo de reconstrucción puede verse afectada por la radiación.

\section{i) Reconstrucción con implantes}

Distintos estudios han demostrado que en pacientes reconstruidas con implantes, la radioterapia:

- Constituye un factor de riesgo para el desarrollo de complicaciones mayores ${ }^{25}$.

- Tiene un impacto negativo significativo en la satisfacción y calidad de vida de las pacientes ${ }^{26}$.

- Se asocia a un mayor riesgo de contractura capsular severa ${ }^{27}$.

Es importante señalar que algunos estudios muestran que pacientes reconstruidas con implantes de forma inmediata que luego reciben radioterapia tienen mayores complicaciones, mayor tasa de reintervención y peor resultado estético que aquellas irradiadas que se reconstruyen de forma diferida con la misma técnica ${ }^{28,29}$. Durante los últimos años ha habido interés creciente en el uso de infiltración de grasa autóloga (lipofilling) en el tejido radiado con el fin de mejorar la calidad del mismo y así posibilitar la expansión y posterior colocación de prótesis de forma más segura y con menos complicaciones ${ }^{30}$.

En contraposición a lo anterior, hay estudios donde la radioterapia no parece incrementar el riesgo de complicaciones de forma significativa y otros en los que se concluye que si bien existe un mayor riesgo de complicaciones, la tasa de contractura capsular $\mathrm{y}$ reintervención es aceptable y que por lo tanto, la reconstrucción con implantes sería una alternativa razonable en pacientes candidatas a radioterapia ${ }^{31,32}$. Por otra parte, otros estudios sugieren que la mayoría de estas pacientes tienen un resultado final bueno a excelente y que mantienen su reconstrucción a los 12 años de seguimiento, aún cuando las tasas de pérdida de implante y de contractura grado IV son significativamente mayores en el grupo irradiado ${ }^{33}$.

\section{ii) Reconstrucción con tejido autólogo}

Existe consenso en que la reconstrucción con tejido autólogo tolera la radioterapia mejor que los implantes ${ }^{34}$. No obstante, los colgajos no se encuentran exentos de riesgo tanto si se realizan sobre un tejido previamente radiado como si se someten a radioterapia post reconstrucción. Algunos estudios sobre el tema muestran que:

- La radioterapia preoperatoria podría ser un factor de riesgo para el desarrollo de complicaciones vasculares intraoperatorias en pacientes sometidas a reconstrucción microquirúrgica ${ }^{35}$. Sin embargo, esta observación no ha sido corroborada por otros autores ${ }^{25}$.

- Comorbilidades como el tabaquismo y diabetes aumentan el riesgo de necrosis grasa y fibrosis en pacientes con radioterapia post reconstrucción ${ }^{36}$.

- La radioterapia post reconstrucción podría asociarse a pérdida de volumen y necrosis grasa del colgajo $^{37}$, aunque nuevamente hay estudios que no muestran dicha asociación ${ }^{38}$.

- Los resultados estéticos son comparables entre pacientes irradiadas y no irradiadas ${ }^{38}$.

Un punto importante a considerar es el intervalo de tiempo entre la radioterapia y la reconstrucción. Mientras un estudio muestra un aumento significativo en la pérdida de colgajos en pacientes reconstruidas a menos de un año de la radioterapia ${ }^{39}$, otros no encuentran tales diferencias, incluso con punto de corte a seis meses ${ }^{40}$. De esta manera, existe actualmente cierta tendencia a reconstruir antes del año $0^{41}$. Otro aspecto a señalar es el desarrollo de nuevos métodos de irradiación que podrían disminuir los efectos deletéreos sobre los tejidos blandos, haciendo por tanto la reconstrucción inmediata autóloga aún más segura ${ }^{42}$.

\section{Matrices dérmicas acelulares}

Durante los últimos años, el uso de matrices dérmicas acelulares se ha incrementado considerablemente en cirugía mamaria tanto estética como reconstructiva. Si bien aún no existen grandes estudios a largo plazo, muchos trabajos han mostrado su relativa seguridad y efectividad lo cual ha llevado a 
Tabla 2. Resumen de matrices dérmicas acelulares disponibles actualmente

\begin{tabular}{|c|c|c|c|c|}
\hline Matriz & Origen & Componentes & Estéril & Indicaciones \\
\hline AlloDerm & $\begin{array}{l}\text { Piel de cadáver } \\
\text { humano }\end{array}$ & $\begin{array}{l}\text { Colágeno, acido hialurónico, } \\
\text { elastina, fibronectina, proteo- } \\
\text { glicanos, canales vasculares }\end{array}$ & No & $\begin{array}{l}\text { Reconstrucción mamaria, pélvica y } \\
\text { cabeza y cuello; reparación de hernia } \\
\text { abdominal }\end{array}$ \\
\hline Strattice & Dermis de porcino & No revelados & Sí & $\begin{array}{l}\text { Reconstrucción mamaria y de pared } \\
\text { abdominal }\end{array}$ \\
\hline DermaMatrix & Piel humana & Colágeno, elastina & Sí & $\begin{array}{l}\text { Reconstrucción mamaria, nasal y párpa- } \\
\text { do inferior; reparación de fisura palatina } \\
\text { y pared abdominal }\end{array}$ \\
\hline SurgiMend & $\begin{array}{l}\text { Colágeno dérmico } \\
\text { de feto bovino }\end{array}$ & Colágeno tipo I y III & Sí & $\begin{array}{l}\text { Reforzamiento de colgajos musculares, } \\
\text { reparación de hernias abdominal, ingui- } \\
\text { nal, diafragmática, femoral, escrotal, } \\
\text { umbilical e incisional }\end{array}$ \\
\hline Veritas & Pericardio bovino & Colágeno & Sí & Reparación de hernia abdominal \\
\hline FlexHD & $\begin{array}{l}\text { Piel de cadáver } \\
\text { humano }\end{array}$ & Colágeno & No & $\begin{array}{l}\text { Reconstrucción mamaria y de pared to- } \\
\text { rácica; reparación de hernia abdominal }\end{array}$ \\
\hline
\end{tabular}

Adaptada de Ibrahim AM, Ayeni OA, Hughes KB, Lee BT, Slavin SA, Lin SJ. Acellular dermal matrices in breast surgery: a comprehensive review. Ann Plast Surg. 2013;70:732-8.

muchos centros a incorporar su uso para reconstrucción mamaria. Actualmente existen distintos tipos de matrices cuyas características principales se resumen en la Tabla $2^{43}$.

Con respecto a las indicaciones, aunque no hay un listado definitivo, las situaciones en donde más se ha descrito el uso de matrices dérmicas son ${ }^{44}$ :

- En reconstrucción inmediata con prótesis, permite crear un bolsillo que cubra completamente la prótesis, especialmente en su polo inferolateral.

- En reconstrucción con expansor-prótesis otorgan mayor soporte a la cobertura muscular. Por otra parte, al usar la matriz se puede evitar la elevación del músculo serrato anterior haciendo la técnica más sencilla.

- En reconstrucción con implantes (directa o en dos tiempos), la matriz ayuda a recrear el surco submamario y el contorno lateral de la mama lo cual puede traducirse en un mejor resultado estético. Por otra parte puede ser utilizada para dar mayor grosor a la cobertura del implante con el fin de evitar irregularidades en la piel.

Dos ventajas importantes a destacar de las matrices acelulares son que podrían reducir la tasa de contractura capsular post reconstrucción y disminuir daños secundarios a la radioterapia ${ }^{45,46}$. Además, en reconstrucción con prótesis directa, su uso no significaría un mayor coste económico ${ }^{47}$. Por el contrario, una de las principales desventajas atribuidas a su uso es que se asocian a un mayor índice de complicaciones como infección, necrosis del colgajo de la mastectomía y seroma ${ }^{48}$. No obstante existen estudios y revisiones que cuestionan esto último ${ }^{49}$. La sociedad Americana de Cirugía Plástica, en sus guías de práctica clínica publicadas recientemente, ponen de manifiesto la variabilidad de la evidencia disponible sobre el uso de matrices dérmicas acelulares en reconstrucción mamaria con implantes y por tanto recomiendan evaluar cada caso de forma particular ${ }^{6}$.

\section{Conclusión}

La reconstrucción mamaria ha experimentado importantes cambios durante las últimas décadas. Los procedimientos basados en el uso de implantes son los más utilizados y con los cuales se obtienen resultados satisfactorios, siempre y cuando se seleccione de forma adecuada a la paciente. Por otra parte, el desarrollo de la microcirugía ha significado una evolución importante en cuanto a las técnicas de reconstrucción autólogas, y aunque existe una variedad de opciones relativamente amplia, los colgajos libres parecen ser la mejor alternativa en pacientes irradiadas o susceptibles de recibir dicho tratamiento. Finalmente, cabe destacar el rol cada vez más importante que están adquiriendo las matrices dérmicas acelulares. Sin dejar de lado el alto índice de complicaciones reportado por algunos autores, su uso parece seguro y además confieren una serie de ventajas importantes en casos de reconstrucción con implantes ya sea en uno o dos tiempos. 


\section{Referencias}

1. http://globocan.iarc.fr/Default.aspx. GLOBOCAN 2012: Estimated Cancer Incidence, Mortality and Prevalence Worldwide in 2012.

2. Departamento de Estadísticas e Información de SaludUnidad de Cáncer, Ministerio de Salud, 2011.

3. Greenberg CC, Lipsitz SR, Hughes ME, Edge SB, Theriault $\mathrm{R}$, Wilson JL, et al. Institutional variation in the surgical treatment of breast cancer: a study of the NCCN. Ann Surg. 2011;254:339-45.

4. Morrow M, Li Y, Alderman AK, Jagsi R, Hamilton AS, Graff JJ, et al. Access to breast reconstruction after mastectomy and patient perspectives on reconstruction decision making. JAMA Surg. 2014;149:1015-21.

5. American Society of Plastic Surgery. 2012 Plastic Surgery Statistics Report. http://www.plasticsurgery. org/Documents/news-resources/statistics/2012-PlasticSurgery-Statistics/full-plastic-surgery-statistics-report. pdf.

6. Alderman A, Gutowski K, Ahuja A, Gray D. Postmastectomy Expander Implant Breast Reconstruction Guideline Work Group. ASPS Clinical Practice Guideline Summary on Breast Reconstruction with Expanders and Implants. Plast Reconstr Surg. 2014;134:648e-55e.

7. D'Souza N, Darmanin G, Fedorowicz Z. Immediate versus delayed reconstruction following surgery for breast cancer. Cochrane Database Syst Rev. 2011; 6:CD008674.

8. Unger JG, Saint-Cyr MS, Schaverien M, Cheng A. Breast Reconstruction. Sel Read Plast Surg. 2014;11:187.

9. Kronowitz SJ. Immediate versus delayed breast reconstruction. Clin Plastic Surg. 2007;34:39-50.

10. Kronowitz SJ, Robb GL. Radiation therapy and breast reconstruction: a critical review of the literature. Plast Reconstr Surg. 2009;124:395-408.

11. Kronowitz SJ, Hunt KK, Kuerer HM, Babiera G, McNeese MD, Buchholz TA, et al. Delayed-immediate breast reconstruction. Plast Reconstr Surg. 2004; 113:1617-28.

12. McCue JD, Migliori M, Cunningham BL. Expanders and Breast Reconstruction with Gel and Saline Implants. In: Hall-Findlay EJ, Evans GR, eds. Aesthetic and Reconstructive Surgery of the Breast. 1st ed. Saunders, Philadelphia, 29-50.

13. Mathes SJ, Alkureishi LW. Secondary breast reconstruction. In: Mathes SJ, editor. Plastic Surgery. Philadelphia: Saunders Elseiver; 2006. p. 1083e174.

14. Ibáñez M, Ibáñez G, Pereira N, Mandiola C, Andino R. Breast reconstruction with pedicled TRAM flap. Rev Chil Cir. 2012;64:40-5.

15. Atisha D, Alderman AK. A systematic review of abdominal wall function following abdominal flaps for postmastectomy breast reconstruction. Ann Plast Surg. 2009;63:222-30.
16. Egeberg A, Rasmussen MK, Sørensen JA. Comparing the donor-site morbidity using DIEP, SIEA or MSTRAM flaps for breast reconstructive surgery: a metaanalysis. J Plast Reconstr Aesthet Surg. 2012;65:147480.

17. Spiegel AJ, Khan FN. An Intraoperative algorithm for use of the SIEA flap for breast reconstruction. Plast Reconstr Surg. 2007;120:1450-9.

18. Howard MA, Mehrara B. Emerging trends in microsurgical breast reconstruction: deep inferior epigastric artery perforator (DIEP) and the superior gluteal artery perforator (SGAP) flaps. Int J Surg. 2005;3:53-60.

19. Schoeller T, Huemer GM, Wechselberger G. The transverse musculocutaneous gracilis flap for breast reconstruction: guidelines for flap and patient selection. Plast Reconstr Surg. 2008;122:29-38.

20. Buchel EW, Dalke KR, Hayakawa TE. The transverse upper gracilis flap: Efficiencies and design tips. Can J Plast Surg. 2013;21:162-6.

21. Allen RJ, Haddock NT, Ahn CY, Sadeghi A. Breast reconstruction with the profunda artery perforator flap. Plast Reconstr Surg. 2012;129:16e-23e.

22. Lee KT, Mun GH. A systematic review of functional donor-site morbidity after latissimus dorsi muscle transfer. Plast Reconstr Surg. 2014;134:303-14.

23. Recht A, Edge SB, Solin LJ, Robinson DS, Estabrook A, Fine RE, et al. Postmastectomy radiotherapy: clinical practice guidelines of the American Society of Clinical Oncology. J Clin Oncol. 2001;19:1539-69.

24. EBCTCG (Early Breast Cancer Trialists' Collaborative Group), McGale P, Taylor C, Correa C, Cutter D, Duane F, Ewertz M, et al. Effect of radiotherapy after mastectomy and axillary surgery on 10-year recurrence and 20year breast cancer mortality: meta-analysis of individual patient data for 8135 women in 22 randomised trials. Lancet 2014;383:2127-35.

25. Berry T, Brooks S, Sydow N. Complication rates of radiation on tissue expander and autologous tissue breast reconstruction. Ann Surg Oncol. 2010;17(Suppl 3):202-10.

26. Albornoz CR, Matros E, McCarthy CM, Klassen A, Cano SJ, Alderman AK, et al. Implant breast reconstruction and radiation: a multicenter analysis of long-term health-related quality of life and satisfaction. Ann Surg Oncol. 2014;21:2159-64.

27. Whitfield GA, Horan G, Irwin MS, Malata CM, Wishart GC, Wilson CB. Incidence of severe capsular contracture following implant-based immediate breast reconstruction with or without postoperative chest wall radiotherapy using 40 Gray in 15 fractions. Radiother Oncol. 2009;90:141-7.

28. Roostaeian J, Pavone L, Da Lio A, Lipa J, Festekjian J, Crisera C. Immediate placement of implants in breast reconstruction: patient selection and outcomes. Plast Reconstr Surg. 2011;127:1407-16.

29. Lee BT, A Adesiyun T, Colakoglu S, Curtis MS, Yueh 
JH, E Anderson K, et al. Postmastectomy radiation therapy and breast reconstruction: an analysis of complications and patient satisfaction. Ann Plast Surg. 2010;64:679-83.

30. Sarfati I, Ihrai T, Kaufman G, Nos C, Clough KB. Adipose-tissue grafting to the post-mastectomy irradiated chest wall: preparing the ground for implant reconstruction. J Plast Reconstr Aesthet Surg. 2011;64:1161-6.

31. McCarthy CM, Mehrara BJ, Riedel E, Davidge K, Hinson A, Disa JJ, et al. Predicting complications following expander/implant breast reconstruction: an outcomes analysis based on preoperative clinical risk. Plast Reconstr Surg. 2008;121:1886-92.

32. Ho AL, Bovill ES, Macadam SA, Tyldesley S, Giang J, Lennox PA. Postmastectomy radiation therapy after immediate two-stage tissue expander/implant breast reconstruction: a University of British Columbia perspective. Plast Reconstr Surg. 2014;134:1e-10e.

33. Cordeiro PG, Albornoz CR, McCormick B, Hu Q, Van Zee K. The Impact of Postmastectomy Radiotherapy on Two-Stage Implant Breast Reconstruction: An Analysis of Long-Term Surgical Outcomes, Aesthetic Results, and Satisfaction over 13 Years. Plast Reconstr Surg. 2014;134:588-95.

34. Barry M, Kell MR. Radiotherapy and breast reconstruction: a meta-analysis. Breast Cancer Res Treat. 2011;127:15-22.

35. Fosnot J, Fischer JP, Smartt JM Jr, Low DW, Kovach SJ 3rd, Wu LC, et al. Does previous chest wall irradiation increase vascular complications in free autologous breast reconstruction? Plast Reconstr Surg. 2011;127:496-504.

36. Albino FP, Koltz PF, Ling MN, Langstein HN. Irradiated autologous breast reconstructions: effects of patient factors and treatment variables. Plast Reconstr Surg. 2010;126:12-16.

37. Mirzabeigi MN, Smartt JM, Nelson JA, Fosnot J, Serletti JM, Wu LC. An assessment of the risks and benefits of immediate autologous breast reconstruction in patients undergoing postmastectomy radiation therapy. Ann Plast Surg. 2013;71:149-55.

38. Crisera CA, Chang EI, Da Lio AL, Festekjian JH, Mehrara BJ. Immediate free flap reconstruction for advanced-stage breast cancer: is it safe? Plast Reconstr Surg. 2011;128:32-41.

39. Baumann DP, Crosby MA, Selber JC, Garvey PB,
Sacks JM, Adelman DM, Optimal timing of delayed free lower abdominal flap breast reconstruction after postmastectomy radiation therapy. Plast Reconstr Surg. 2011;127:1100-6.

40. Momoh AO, Colakoglu S, de Blacam C, Gautam S, Tobias AM, Lee BT. Delayed autologous breast reconstruction after postmastectomy radiation therapy: is there an optimal time? Ann Plast Surg. 2012;69:14-18.

41. Kronowitz SJ. Current status of autologous tissuebased breast reconstruction in patients receiving postmastectomy radiation therapy. Plast Reconstr Surg. 2012;130:282-92.

42. Haviland JS, Owen JR, Dewar JA, Agrawal RK, Barrett J, Barrett-Lee PJ, et al. START Trialists' Group. The UK Standardisation of Breast Radiotherapy (START) trials of radiotherapy hypofractionation for treatment of early breast cancer: 10-year follow-up results of two randomised controlled trials. Lancet Oncol. 2013;14:1086-94.

43. Ibrahim AM, Ayeni OA, Hughes KB, Lee BT, Slavin SA, Lin SJ. Acellular dermal matrices in breast surgery: a comprehensive review. Ann Plast Surg. 2013;70:7328.

44. Spear SL, Parikh PM, Menon NG. Acellular dermisassisted breast reconstruction. In: Spear SL, Willey SC, Robb GL, Hammond DC, Nahabedian MY, eds. Surgery of the Breast: Principles and Art. $3^{\text {rd }}$ edition. Philadelphia: Lippincott Williams \& Wilkins 2011;406-11.

45. Hester TR Jr, Ghazi BH, Moyer HR, Nahai FR, Wilton M, Stokes L. Use of dermal matrix to prevent capsular contracture in aesthetic breast surgery. Plast Reconstr Surg. 2012;130(5 Suppl 2):126S-36S.

46. Moyer HR, Pinell-White X, Losken A. The effect of radiation on acellular dermal matrix and capsule formation in breast reconstruction: clinical outcomes and histologic analysis. Plast Reconstr Surg. 2014;133:21421.

47. Jansen LA, Macadam SA. The use of AlloDerm in postmastectomy alloplastic breast reconstruction: part II. A cost analysis. Plast Reconstr Surg. 2011;127:2245-54.

48. Israeli R. Complications of acellular dermal matrices in breast surgery. Plast Reconstr Surg. 2012;130:159S-72S.

49. Clemens MW, Kronowitz SJ. Acellular dermal matrix in irradiated tissue expander/implant-based breast reconstruction: evidence-based review. Plast Reconstr Surg. 2012; 130:27S-34S. 